\title{
Understanding the perceptions of UK COVID-19 contact tracing app in the BAME community in Leicester
}

Simisola Akintoye

Centre for Law, Justice and Society, De Montfort University, Leicester, UK

George Ogoh

Centre for Computing and Social Responsibility, De Montfort University, Leicester, UK

\section{Zoi Krokida and Juliana Nnadi}

Centre for Law, Justice and Society, De Montfort University, Leicester, UK, and

Damian Eke

Centre for Computing and Social Responsibility, De Montfort University,

Leicester, UK

\begin{abstract}
Purpose - Digital contact tracing technologies are critical to the fight against COVID-19 in many countries including the UK. However, a number of ethical, legal and socio-economic concerns that can affect uptake of the app have been raised. The purpose of this research is to explore the perceptions of the UK digital contact tracing app in the Black, Asian and Minority Ethnic (BAME) community in Leicester and how this can affect its deployment and implementation.
\end{abstract}

Design/methodology/approach - Data was collected through virtual focus groups in Leicester, UK. A total of 28 participants were recruited for the study. All participants are members of the BAME community, and data was thematically analysed with NVivo 11.

Findings - A majority of the participants were unwilling to download and use the app owing to legal and ethical concerns. A minority were willing to use the app based on the need to protect public health. There was a general understanding that lack of uptake will negatively affect the fight against COVID-19 in BAME communities and an acknowledgement of the need for the government to rebuild trust through transparency and development of regulatory safeguards to enhance privacy and prevent misuse.

Originality/value - To the best of the authors' knowledge, the research makes original contributions being the first robust study conducted to explore perceptions of marginalised communities, particularly

(C) Simisola Akintoye, George Ogoh, Zoi Krokida, Juliana Nnadi and Damian Eke. Published by Emerald Publishing Limited. This article is published under the Creative Commons Attribution (CC BY 4.0) licence. Anyone may reproduce, distribute, translate and create derivative works of this article (for both commercial and non-commercial purposes), subject to full attribution to the original publication and authors. The full terms of this licence may be seen at http://creativecommons.org/ licences/by/4.0/legalcode

Funding acknowledgements: This work was supported by the European Union's Horizon 2020 Human Brain Project Framework Programme for Research and Innovation under the Specific Grant Agreement No. 945539 (HBP SGA3).

This work was also supported by De Montfort University COVID-19 Special Research Fund.
Received 30 June 2021 Revised 26 July 2021 Accepted 26 July 2021 
JICES

19,4

BAME which may be adversely impacted by the deployment of the app. By exploring community-based perceptions, this study further contributes to the emerging citizens' perceptions on digital contact tracing which is crucial to the effectiveness and the development of an efficient, community-specific response to public attitudes towards the app. The findings can also help the development of responsible innovation approaches that balances the competing interests of digital health interventions with the needs and expectations of the BAME community in the UK.

Keywords Privacy, Public health, COVID-19, Data protection, Contact tracing, BAME in the UK

Paper type Research paper

\section{Background}

The United Kingdom (UK) accounts for more than 150,000 deaths of the over 3 million COVID-19 related deaths recorded globally as of 25 April 2021. Evidence from both academic literature and public health reports demonstrate that people from the black, Asian and minority ethnic groups (BAME) have had the highest rate of infection and mortality (PHE, 2020a, 2020b; Otu et al., 2020; ONS, 2020). Beyond ethnicity, age, sex and geographical location have also been highlighted as having impacts on the risk of infection, symptomatic presentation and ultimately death rates. Similar statistics showing a disproportionate number of cases in black and minority communities have also emerged on a global level including in the USA (Bäcker, 2020; Yancy, 2020) and other countries (Bowleg, 2020).

Whilst the disparities in statistics illuminate varying underlying inequalities and intersectionality that can yield poorer health outcomes for COVID-19 in BAME communities across the world, in the UK, there is an urgency to understand the complex factors behind the statistics. The UK Research and Innovation (UKRI) and the National Institute for Health Research are funding about six research projects in 2020 to improve our understanding of the links between COVID-19 and ethnicity and to develop measures to mitigate the disproportionate death rates (UKRI, 2021). Clearly, the spread of COVID-19 has exposed societal flaws, inequalities and inconsistencies with BAME communities and on a wider scale, necessitated the adoption of novel strategies for curbing the spread of the infection.

Among the earliest strategies adopted by a number of countries was digital contact tracing. Contact tracing is a well-established and sophisticated disease control measure that aims to follow the chains of infection to uncover cases. Common controls combined with contact tracing include vaccination (Anderson and May, 1992; McLean and Anderson, 1988), pre-emptive culling (Woolhouse and Donaldson, 2001) and public health campaigns (Eames, 2007) which have been successfully used to tackle and even eradicate the spread of diseases such as tuberculosis, sexually transmitted diseases and smallpox (Bazin, 2000). However, it is now believed that targeting such control measures through a multi-stage process is likely to lead to a more radical and effective increase in the treatment and management of diseases. Traditional contact tracing - which relies on a team of public health workers who manually, via interviews over the phone, trace possible contacts of a patient who has tested positive to an infectious disease - was successfully used to curb the spread of Ebola. Although the mortality (death) rate of Ebola is higher than COVID-19, the reproduction rate of the latter is higher which implies that it spreads faster in a population (Fox, 2020) and the prevalence of asymptomatic cases is also higher in COVID-19 (Zhang and Jain, 2020).

In view of these, digital tracing technologies have been heralded as critical as case management in the fight against the coronavirus pandemic. The underlying logic here is that, given their unique characteristics, digital technologies can help to: identify more contacts than traditional methods of contact tracing, simplify the electronic capture and management of data on patients and contacts and effectively integrate workflows with surveillance systems. Some of the available frameworks include proximity tracing, location 
tracking (Holmes, 2020), GEO-QR code tagging, ultrasound (Novid, 2020) and CCTV with facial recognition. Proximity tracing, deployed primarily in Europe, involves the measurement of signal strength to determine whether two devices (such as smartphones) were close enough that there is a significant risk of transmission of the virus from one user to the other. (Crocker et al., 2020; WHO, 2020a). The primary purpose of this system is to provide a mechanism that alerts users who may have been exposed to the virus, serving as a trigger for a precautionary intervention (usually testing or self-isolation) recommended by a public health authority (Troncoso et al., 2020).

On 12 April 2020, the UK government announced that the digital innovation unit of the NHS, NHSx, was developing a contact tracing technology called the NHS COVID-19 app to support the fight against COVID-19. This proximity tracing tool was designed based on a centralized architecture where the personal data collected through the App will be controlled by the public health authority. Even though this model mainly follows the Pan-European Privacy-Preserving Proximity Tracing Initiative protocols (Cooper et al., 2020), there was an understanding in the information and communication technology (ICT) community that this might not be the most privacy-preserving framework. Under this model, the collected data are anonymised and uploaded to a remote server, managed by the NHSx, where contacts are matched, risk analysis conducted and notifications sent out to possible contacts of identified patients.

The choice of this model saw the UK diverge from a favoured decentralized approach (such as the one jointly designed by Apple and Google) where personal data are controlled by individuals only on personal devices (Li and Guo, 2020). Under this model, ID codes generated during "digital handshakes" are stored in a decentralized way and are regularly downloaded to the users' phone allowing them to check for matching codes. These codes change regularly, making re-identification of data subjects more difficult. Unlike the centralized system, the matching process does not happen in a central server and the AppleGoogle partnership seeks to enhance interoperability under the decentralized model.

The UK started piloting its centralized proximity tracing app on the Isle of Wight on 5 May 2020, and findings from this trial put the reliability of the app into serious question. Following reported technical flaws (including the app only able to detect $4 \%$ of iPhone and $75 \%$ of android phones) and identifiable concerns from the ICT community, the UK government announced in June, that it was switching to the Apple-Google's Bluetoothoriented exposure notification application programming interfaces (API) model In abandoning its bespoke app, the UK government moved to a decentralized "privacy-centric" technology that was launched in the fall of 2020. It should be pointed out that there have been other developments (e.g. concerns over pinging) since the launch of the contact tracing app that was not considered in this paper. This digital tracing technology is not proposed to be a replacement for traditional methods and is only effective when integrated into an existing public health system (Gillmor, 2020). Thus, the NHS COVID app is part of the UK's test and trace system that includes traditional methods of contact tracing. The most crucial elements of the implementation of digital contact tracing tools include "community engagement and public support; careful planning and consideration of local contexts, communities and cultures" (WHO, 2020b).

However, the potential effectiveness of the app largely depends on wide-scale adoption (downloading and using) which in turn depends on a lot of other factors. Considering the disproportionate impact of COVID-19 on UK BAME communities, minority communities are expecting intervention strategies, including proximity tracing technologies to contribute positively to the fight against the virus. In view of this, it becomes critical to understand the perceptions of the UK BAME community on the legal, socio-cultural and economic impacts 
JICES

19,4

of this technology which can affect its implementation. In 2011, Leicester was found to have a significantly higher than average population of BAME (third highest) when compared to the average in England (Leicester City Council, 2011). It is therefore imperative to consider the impact of digital technologies on marginalised communities.

In view of the above, this study examines the perceptions of the BAME community in Leicester regarding the UK COVID-19 tracing app. The aim of the paper is to contextualize the perspectives of the BAME community, whether they plan to use it and what factors will influence their decisions. These perspectives can contribute to a wider understanding of the deployment, as well as the reception and adoption of the technology which can ultimately shape future use of digital contact tracing in the UK, especially for the BAME communities.

\section{Data collection}

A total of six virtual focus group sessions were organised with 28 participants resident in Leicester from 22 to 27 June 2020. Participants were recruited via a registration system and advertised through identified community networks in Leicester. Each of these sessions had between 5 and 10 participants aged 18 and above. Owing to current restrictions from COVID, the focus group sessions were conducted online using the virtual conferencing tool, Zoom and the average duration of each focus group was 60 min. All participants were members of the BAME community. To ensure a sufficient level of cultural and gender diversity, each focus group consisted of both men and women and participants from more than one ethnic minority group. It is important to state that the focus group discussions were organized before the app was officially launched in the UK and the research received ethics approval from the University's ethics approval committee. The researchers, who served as moderators of these sessions, developed detailed guidelines with defined responsibilities so as to facilitate structured and consistent discussions. Focus group discussions are valuable tools that emphasize holistic descriptions of knowledge and experiences. O Nyumba et al. (2018) observed that focus groups are frequently used to gain an in-depth understanding of social issues. It has notably been applied in studies to demonstrate a particular social group's common values and cognitions (Miltgen and PeyratGuillard, 2014). Because this study focuses on the BAME community with some unique characteristics, focus groups are succinct because they "capitalize on the interaction within a group to elicit rich experiential data" (Asbury, 1995, p. 414). Its popularity as a method in socio-cultural research is informed by its sensitivity to cultural variables (Miltgen and Peyrat-Guillard, 2014). The focus group discussions were guided by a set of questions around the theme of the research. The same guide was used for all the sessions moderated by the same researchers and all the sessions included both male and female participants. All the participants had some level of familiarity with digital technology and responses were based on their knowledge and experiences. Participants were engaged throughout the sessions with very lively conversations. Every participant had an opportunity to contribute fully with no domination from others. Each participant was also encouraged to comment.

The choice of Leicester was influenced by a number of reasons: Firstly, according to information from the Office for National Statistics (ONS, 2011), Leicester is one of top ten cities with the highest number of BAME population (close to $50 \%$ of the city's population recorded as BAME). Secondly, in June, 2020 Leicester became the first UK city to be put under a local lockdown following what was described by the UK Health Secretary as an "outbreak" of COVID-19. Participants were recruited via a registration system as advertised through identified community networks in Leicester. Whilst a majority of the BAME community in Leicester are Asians, only a small percentage participated in the research 
(Table 1). The scientific implication of this is detailed in the limitations and future research section.

\section{Analysis}

This research adopted a thematic analysis method. Transcribed and anonymised data from the focus groups were analysed in NVivo software version 11 and relevant themes were inductively identified. The research team comprised five members. The initial analysis was done by two people whilst a third person read through the transcripts to identify recurrent themes to be used as codes. This resulted in the identification of seven general themes. These were reviewed and validated by the final two members who identified additional four themes. Following this, all researchers reviewed the eleven themes against the data, modified and validated the themes. These were then categorized into four high level groups: legal and ethical concerns, socio-economic concerns, technical concerns and recommendations. This analysis focused solely on the transcribed texts and does not include differences in body language and how other variables like age and gender affected the responses.

\section{Findings}

The findings show that the majority of the participants were not willing to download and use the contact tracing app. These participants' concerns centred on legal and ethical considerations, socio-economic factors and technical concerns. The group of participants who were undecided expressed similar concerns but were hoping for some changes to be convinced. However, three participants expressed their willingness to download the app based on the positive impact it might have for public health, particularly for the BAME community. Additional themes that emerged across all focus group sessions are grouped under recommendations. In the participants' perspectives, the development and roll out of the app should have been different. These perceptions are detailed in the following sections.

\section{Legal and ethical concerns}

\section{Privacy}

Majority of the participants expressed concerns bordering on privacy and security of personal data. The concerns were for both the centralized and decentralized approaches to the app. There was a consensus among the participants who believed that it was impossible to guarantee anonymity of the data. For example, one participant pointed out the difficulty of "guaranteeing anonymity of data in the age of artificial intelligence and deep learning [...] as the system was developed to identify you and your contacts?"

Another participant also expressed similar concerns related to the difficulty of anonymisation which relates to the issue consent in the statement below:

It is misleading to say that it is completely anonymous.

\begin{tabular}{lc}
\hline Ethnicity & No. of participants/percentage (\%) \\
\hline Black & $22(78.57)$ \\
Asian & $4(14.29)$ \\
Other minority ethnic groups & $2(7.14)$ \\
Total & 28
\end{tabular}

UK COVID-19 contact tracing app \\ 525}



of the possibility of the government or other organizations accessing these data and using it for other purposes. One participant asked:

[... ] wouldn't this again lead to another form of profiling? Because I've already seen people being profiled with information from non-related systems and not being able to get loans because of probably their background. I think we are already a community that is very sceptical [...] because of the structured systems and the inequalities that we have faced.

In some cases, such concerns also stem from unique circumstances such as immigration status of some members of the BAME community as expressed by one participant:

For the fact that the identifiable personal information can be assessed and used is worrying. Especially for those who may have immigration issues, they will be worried about information about where they live, where they work and the people they associate with, being shared in future with police, with health organizations and with immigration officials. So, these are some of the areas that could pose some challenges for the BAME Community.

Some of these privacy concerns were also informed by the fact that the nature and scope of data collection were unclear as thus started:

I am concerned about the privacy of my data because I am not too clear about what data they are going to collect from us or what data they are going to share.

\section{Lack of trust}

Another recurring concern for those unwilling to download and use the app or those undecided about usage was the issue of lack of trust in the institutions, both public and private, involved in the app. One participant captured this sentiment in the following sentences:

I just feel like there's a lot of unanswered questions and especially about how they plan to use our data once this whole thing is over. I know they have mentioned that it will be completely deleted. But do we in our communities trust the government, the tech organizations or other institutions involved in this app? Look at what is happening around the world with black and brown communities. What level of trust do we have in these rigged institutions?

Many of the participants acknowledged that this concern is not just about the technology but the long-standing relationship between the BAME communities and public institutions. A participant had this to say:

Yes, I agree that this is an issue about the technology or the app in particular. It is the long running issues of trust between the public systems and our communities. I guess the government did not consider this because For ethnic minorities, we need a little bit more information and persuasion for us to be able to trust or believe that this actually is going to work for us and it's going to help us because of the long standing issues culturally, or in terms of related to the structural inequalities that we face.

\section{Possibility of misuse}

There was a strong feeling among the participants that there are underlying objectives of the app that the government is not making public. As one participant puts it:

There is a high risk of this app being misused by government agencies and other organizations especially Apple and Google that do not have the best reputations already with misuse of 
technologies around the world and I believe that members of our communities will suffer the most if this happens.

Although this sentiment is not based on evidence, participants presented it as the overwhelming feeling among members of BAME in Leicester. A participant captured it in this way:

In relation to our communities, I agree with the sentiment that this app can be used for improved systematic surveillance or other racial profiling activities. I read on the internet the other day

about the disproportionate way ethnic minorities were being fined for lockdown breaches. I know this fear is based on the historical relationship between government agencies and our communities but that is how we feel and what we believe.

\section{Lack of transparency/lack of adequate information}

Some of the participants believed that there was a general lack of transparency surrounding the development and proposed implementation of the app which affects their perspective on it. There was a consensus among all the participants that the information made available about the meaning, nature and scope of the digital app was not sufficient to convince them of the intentions of the government. Following spates of misinformation online related to COVID-19, the participants felt that government agencies have not done enough to mitigate people's fears and concerns. A participant responded thus:

The response of some stakeholder groups towards the proposed centralized NHSx model and the subsequent change of mind makes this whole app thing suspect. There is something the government is not saying about their aims and objectives with this app. The issue of transparency definitely affects my perception of whatever app that is rolled out. The UK government was not transparent about the negatives of the centralized system and allowed Apple and Google to be on the side of the people as regards privacy, by the introduction of the decentralized privacy-centric system.

Some also pointed to the lack of consistent information coming from the government as a big concern in these words:

Information we are receiving is not consistent. Today, it is the government saying that they will build the app without the Apple and Google API, the next day, they are saying they used the API concurrently as they were developing the first app. We are as confused as they are. This does not make me confident in the app.

\section{Socio-economic concerns}

A number of issues were raised by the participants that border on social and economic concerns. One of these concerns is the possibility of discrimination against those who refuse to use the app. There was also a general belief that majority of people from BAME communities will not download and use the app which will result in scapegoating or discrimination and which may subsequently have economic consequences. A participant described it this way:

The BAME Community may be rejected by employers based on this app which will lead to financial difficulties which will impact on their economic status and the kind of house they live, the kind of education they give to their children and their access to health and well-being.

This seems like a misconception that stems from historical experiences of people from these communities. 
JICES

19,4

\section{Possibility of scapegoating and discrimination}

The possibility of blaming continuous spread of the virus on the people who refuse to download and use the app is also another concern that could lead to possible scapegoating. There was also the feeling that making the app a prerequisite or making it a digital passport for accessing public places or employment facilities can lead to unnecessary discrimination. The following quote captures this perspective:

It seems [that] this might become part and parcel of our lives. If we refuse to use it as is happening in our communities, we can be made scapegoats and blamed for other people getting it. It might also be mandatory to have this app before you access public buildings or transport (such as trains or buses). Even employers might demand it. I can see how this can cause discrimination.

\section{Technical concerns - the impact of false positives/negatives}

The participants raised technical concerns bordering on the reliance of this app on selfreporting of symptoms which can lead to false positives alerts or false negative impressions. One participant noted that in addition to mistrust about the overall effectiveness of the app, there were additional concerns about the negative user experiences involved in the app usage, such as battery drainage and potential unwanted notifications which have restricted their willingness to download the app. False positives might mean a greater number of people being asked to stop work and self-isolate with the effect being the worsening of socioeconomic problems. A participant put it this way:

I have read that false positives or negatives from self-reporting of symptoms as well as the tests can make this app to tell you to self-isolate when you are not supposed to. This will affect my work and my income. This is one of the reasons why I will not be using the app.

\section{The imperative to protect public health}

The participants who intended to use the app pointed out that their underlying motivation is for public health protection. As these participants observed, ignorance may be part of the reasons why people from BAME communities will not use the app:

The level of uptake of this app will really have a significant impact on the BAME Community. Most of them will not download the app because people may be ignorant of the impact it will have on our community that is disproportionately affected by this virus.

\section{Recommendations}

In addition to providing the underlying motivations or factors that will influence the uptake of this app, the participants articulated a number of suggestions that could make the implementation of the app or subsequent digital measure of disease control effective in BAME communities in the UK.

\section{Rebuilding trust}

Majority of the participants believed that this app will not be welcomed in the BAME community unless it is made mandatory. This is as a result of long seated mistrust of the government who are mostly blamed for the socio-economic inequalities giving rise to the disproportionate COVID-19 cases and fatalities. Therefore, there was a near consensus that the government should put in more resources into rebuilding trust in the BAME community to improve the acceptability of the technology. The participants called on the government to engage with more BAME community leaders and initiate public campaigns aimed at 
reassuring these communities that this technology will not target them and will not be misused in any way. This will allay people's fears and imbue some form of trust that will help the overall acceptance of the technology in communities where it is needed the most. Members of the BAME community will have to be able to trust that their data will securely be processed and will not be used in any way to discriminate against them or unjustifiably target them.

\section{Adequate and transparent information}

There was a consensus among the participants that the initiative was not transparent enough and that there should be more transparency. The initial government proposed centralized system was shrouded in uncertainties, and there was no clear information on how and what the data will be used for in the future. The current decentralized plan is also not so transparent. The role of Apple and Google, the type of data involved and potential uses of the data are not clear enough. This concern is supported by recent reports about the Apple and Google API. The government must commit to full transparency on the implementation of the technology. It is important to provide clarity into government decision-making, how decisions with potentially life-altering ramifications are made. Credibility and legitimacy of government decision-making are crucial elements at this time of national crisis.

\section{Regulation}

The participants called for a clear regulation or policy to prevent misuse or dual use of concern. Such policy and a clear path for enforcement will reassure members of the BAME community in some ways. Even though the General Data Protection Regulation (GDPR)'s expansive scope and its principle-based approach offer a functional blueprint for system design that is compatible with fundamental rights (Bradford et al., 2020), it does not seem to provide specific protection against bias and discrimination and other forms of misuse of this technology against the BAME community. In June 2020, the UK Medicines and Healthcare products Regulatory Agency developed guidance for manufacturers, members of the public and professional users called the "Regulatory status of software (including apps) used in the diagnosis, treatment and management of patients with coronavirus" (MHPRA, 2020). Apple and Google also have their requirements. App developers, however, have discovered loopholes in these regulations which present the challenge of helping users to understand which apps are potentially unsafe to use as well as ensuring that they understand the right information about the strengths and weaknesses of the app before downloading and using it. This calls for stronger regulation that protects all users but most especially members of the BAME community.

\section{Critical discussion}

These findings are intricately linked but pervading perceptions of the UK COVID-19 contact tracing app in the BAME community in Leicester. However, it is worth pointing out that some of these concerns such as privacy, lack of transparency, possibility of misuse and technical concerns are not particularly unique to the BAME community. For example, based on current research, Davis (2020) indicates there are other groups of people who would likely avoid contact tracing apps because of similar concerns. These groups include undocumented migrants, the elderly and members of groups like LGBT who are worried about stigmatisation. Nevertheless, the participants cited the historical mistrust in the government, feelings of racism and inequalities and the evident disproportionate profiling by the police as reasons for their heightened concerns. The participants expressed acute 
JICES

19,4

fears, of what the data collected can be used for, among minority communities who have often "been undertreated or abused through the medical system," as well as "undocumented immigrants who fear punitive measures should they present themselves at a clinic or hospital" (Berger et al., 2020). It must also be mentioned that this research was conducted at the middle of a global outcry and protests against the unlawful killing of a black man (George Floyd) by the police in the USA. Experiences of injustice, systemic surveillance and marginalization were given as factors that shaped the participants' perceptions and will shape the level of uptake in BAME communities. This corroborates the findings of a global survey conducted in the UK, the USA, Italy, Germany and France which revealed that people who have low trust in their national governments are more hesitant to install the app on their phones (Altmann et al., 2020).

Participants generally feared increased targeting stemming from function creep through a repurposing of information collected via the app. This is a justifiable fear, especially as it has been noted that "once data has been collected, even for the best reasons, it is not inconceivable that the data is used for unsavory or even terrifying reasons" (Claypoole, 2020). For instance, changes in law and their reinterpretation by courts following $9 / 11$ in the USA contributed to the weakening of civil rights and a shift in the balance between police surveillance authority and civil privacy protection (Bloss, 2007). Lack of adequate information was also highlighted as an additional reason not to trust the intentions of the government. Evidence suggests that users refrain from downloading and using apps when data collection methods, scope and usage are unclear (Almuhimedi, 2017; Chitkara et al., 2017). A telling example is the case of Facebook where it has been reported that the social media platform harvested data from users' mobile app cameras and used them for other services (Goode, 2018). Betzing et al. (2020) also found that mobile app users are more likely to make informed decisions when transparent information about data processing is provided. Transparency is also one of the principles of data protection. According to Recital 58 of the EU GDPR, this principle "requires that any information addressed to the public or to the data subject be concise, easily accessible and easy to understand, and that clear and plain language and, additionally, where appropriate, visualization be used.” In this case, the participants felt that the government who should assume data controllership as regards the app did not provide all the information related to the processing as required by article 13 of the GDPR.

There was also a general feeling that members of the BAME community are already facing extensive government surveillance and are more vulnerable to risks. There could be an underlying use which can lead to more socio-economic and political disadvantage of BAME. A typical example presented by the participants is the case of people in the BAME community without legal documents in the UK who will not be keen to use the technology because they will feel that this can be used to profile them and ultimately depot them. Some of the participants mentioned recent scandals like the Cambridge Analytica and the UK police use of facial recognition as examples of how their data and this technology can be misused. The implication of the latter has been documented in recent reports such as the ICO report on how the police use facial recognition technology in public places (ICO, 2019), The London Policing Ethics Panel final report on LFR (London Policing Ethics Panel, 2019), Cardiff University's evaluation of South Wales Police's use of automated facial recognition (Davies et al., 2018), Essex University's independent report on the Metropolitan Police Service's trial of LFR (Fussey and Murray, 2019), The Home Office's Biometrics and Forensics Ethics Group Facial Recognition Working Group's interim report (Biometrics and Forensics Ethics Group Facial Recognition Working Group, 2019) and Big Brother Watch's "Face Off report (Big Brother Watch, 2018). All these reports confirmed that facial 
recognition used by Police in the UK discriminated against the BAME because of what is referred to as algorithmic bias (Buolamwini and Gebru, 2018). Ultimately, this could lead to wrongful arrest of those from BAME owing to a "false positive." The contact tracing technology is based on AI tools and resources. There have also been reported allegations of racial profiling during the lockdown searches against London police (Quinn and Perraudin, 2020).

Similarly, participants were concerned that the app could have financial impact on their socio-economic lives. They painted a scenario where because of a misuse of data, potential employers are left unwilling to hire individuals that have been affected by COVID-19 or individuals that do not have the app. This understanding has been accentuated in current academic scholarship. For instance, Scassa et al. (2020) have noted that "employers may refuse to allow employees to return to work, or businesses may deny access to individuals who cannot demonstrate that they are using the app." This means that individuals' professional development and economic progress might be restricted because they did not download the app or because their personal data have been misused. This is also in line with recent cases of "pinging" where the app has been subject to operational errors. For instance, it has been reported that many people have erroneously been pinged by the app to selfisolate because the Bluetooth signal of the phone has been strong enough to pass through walls. Therefore, without being in contact with a COVID-19 case, people were asked to shield whilst a shortage of staff have been observed (The Guardian news, 2021). Further, both the participants there were undecided and those that were certain that they were not going to use the app echoed similar sentiments. Although these perspectives might be informed by misconceptions of the app, they contribute to attitudes towards the app. This is consistent with some research which has shown that although "mental models" of technical and legal concepts in users are often incomplete or inaccurate, they play a significant role in the willingness of potential users to begin contact tracing (Simko et al., 2020). However, whilst a general apathy towards safety and privacy might in some cases not hinder the adoption and usage of an app, when coupled with distrust and fear, it could severely hinder its uptake.

Notably the participants were not unanimous in stating their unwillingness to download and use the app. For the $10.7 \%$ of the participants who expressed their willingness to use the app, the overarching concern bothered on the implications of not using the app. For these people, there was a sense that there is a moral imperative to download and use the app because it will have a positive impact on the infection rate in the BAME community. And considering that members of BAME are disproportionately affected by COVID-19, there was a fear that ignorance and misconceptions will affect uptake which will negatively affect infection and death rates in these communities. Nevertheless, all the participants agreed that the uptake of this app will be very low in BAME communities which will be consequential in the fight against the disease in these communities in particular and among the general UK population. That is why the recommendations (making efforts to rebuild trust, providing adequate and transparent information and the establishment of clear regulations) proposed by the participants are very critical, not only for the COVID-19 contact tracing app but for other similar digital solutions for public health emergencies.

\section{Limitations and future research}

This study has a number of limitations. First is methodological limitation. The outcome of this study is based on an interpretive approach; findings are therefore open to multiple interpretations because of ambiguities inherent in language perception and understanding of sociotechnical imaginaries. One way this problem may have been overcome is to use 
JICES

19,4

multiple data sources to provide triangulation, for example, by also using a quantitative approach to back up the findings of the research. Such an approach will be applied in future as a follow on of this research. Nevertheless, it should be pointed out that much effort was put into ensuring that a good understanding of the meanings of the participants' responses was obtained by constantly going back and forth between individual sentences and whole transcripts in an attempt to grasp true meaning.

Secondly, this study was conducted in Leicester; therefore, participants form a small percentage of the overall UK BAME population. Furthermore, it must be stated that within Leicester, only a small percentage of Asians participated in the research. Whilst findings provide a good indication of the perception of the BAME communities on the app, results can only be applied to other BAME communities in the UK with the contextual dynamics of participation in mind. To overcome this, studies with more representative samples and regional spread would have to be conducted. Further qualitative study to understand the complex and nuanced perceptions within the BAME community or how gender, age and employment influence these perceptions may be needed.

\section{Conclusion}

This study explored the perceptions of UK BAME communities of the contact tracing app and examined the legal, ethical and socio-cultural issues related to the app and how this affects already marginalised communities in the UK. The research makes original contributions being the first robust study conducted to explore perceptions of marginalised communities which may be adversely impacted by the deployment of the app. The study further contributes to the emerging citizens' perceptions on digital contact tracing which is crucial to the effectiveness and the development of an efficient, community-specific response to public attitudes towards the app. The paper concludes that there is a plethora of concerns that can impact the acceptability and effectiveness of the UK contact tracing app in the BAME communities. Contact tracing apps have a significant role to play in the global fight against COVID-19 as the world begins to come to terms with the disease by building a resilient health system. However, it is important that public health measures to target pandemics such as COVID-19 are people-centric to gain trust; therefore, historical mistrust of the government needs to be adequately addressed.

Furthermore, it is also crucial that marginalized communities are assured that digital technologies used as health interventions tools will not negatively impact the public. This calls for development of responsible innovation models that emphasize community-based engagements for new digital technologies introduced for public healthcare interventions. Transparent engagement of BAME community leaders can help in identifying specific community concerns. Such community-specific considerations can increase faith in the process of tech development and implementation. This aligns well with the principle of responsible innovation that will not only involve a robust privacy by design approach but also the consideration of socio-cultural and ethical concerns.

\section{References}

Almuhimedi, H. (2017), "Helping smartphone users manage their privacy through nudges".

Altmann, S., Milsom, L., Zillessen, H., Blasone, R., Gerdon, F., Bach, R., Kreuter, F., Nosenzo, D., Toussaert, S. and Abeler, J. (2020), "Acceptability of app-based contact tracing for COVID-19: cross-country survey study", JMIR MHealth and Uhealth, Vol. 8 No. 8, p. e19857.

Anderson, R.M. and May, R.M. (1992), Infectious Diseases of Humans: Dynamics and Control, Oxford University Press. 
Asbury, J.-E. (1995), “Overview of focus group research”, Qualitative Health Research, Vol. 5 No. 4, pp. 414-420.

Bäcker, A. (2020), "Why COVID-19 may be disproportionately killing African Americans: black overrepresentation among COVID-19 mortality increases with lower irradiance, where ethnicity is more predictive of COVID-19 infection and mortality than median income (SSRN scholarly paper no. ID 3571699)", Social Science Research Network, Rochester, New York, NY, doi: 10.2139/ssrn.3571699.

Bazin, H. (2000), The Eradication of Smallpox, Elsevier.

Berger, Z.D., Evans, N.G., Phelan, A.L. and Silverman, R.D. (2020), Covid-19: Control Measures Must Be Equitable and Inclusive, British Medical Journal Publishing Group.

Betzing, J.H., Tietz, M., Vom Brocke, J. and Becker, J. (2020), “The impact of transparency on mobile privacy decision making", Electronic Markets, Vol. 30 No. 3, pp. 607-625.

Big Brother Watch (2018), "Face off: the lawless growth of facial recognition in UK policing", available at: Bigbrotherwatch.org.uk

Biometrics and Forensics Ethics Group Facial Recognition Working Group (2019), Ethical issues arising from the police use of live facial recognition technology (Interim Report), UK Home Office, London.

Bloss, W. (2007), "Escalating US police surveillance after 9/11: an examination of causes and effects", Surveillance and Society, Vol. 4 No. 3, p. 4.

Bowleg, L. (2020), "We're not all in this together: on COVID-19, intersectionality, and structural inequality", American Journal of Public Health, Vol. 110 No. 7, pp. 917-917, doi: 10.2105/ AJPH.2020.305766.

Bradford, L., Aboy, M. and Liddell, K. (2020), “COVID-19 contact tracing apps: a stress test for privacy”, The GDPR, and Data Protection Regimes. J. Law Biosci, Vol. 7, doi: 10.1093/jlb/lsaa034.

Buolamwini, J. and Gebru, T. (2018), "Gender shades: intersectional accuracy disparities in commercial gender classification", Proceedings of the 1st Conference on Fairness, Accountability and Transparency, in Proceedings of Machine Learning Research, Vol. 81, pp. 77-91, available at: https://proceedings.mlr.press/v81/buolamwini18a.html (accessed 30 November 2021).

Chitkara, S., Gothoskar, N., Harish, S., Hong, J.I. and Agarwal, Y. (2017), "Does this app really need my location? Context-aware privacy management for smartphones", Proceedings of the ACM on Interactive, Mobile, Wearable and Ubiquitous Technologies, Vol. 1 No. 3, pp. 1-22.

Claypoole, T. (2020), "COVID-19 privacy protection and persecuted minorities", Lexology, available at: www.lexology.com/library/detail.aspx?g=386a141e-6601-4879-9170-5f04f10088e1 (accessed 30 April 2021).

Cooper, D., Quathem, K.V. and Meneses, A.O. (2020), "COVID-19 apps and websites - the 'PanEuropean privacy preserving proximity tracing initiative' and guidance by supervisory authorities. Priv", available at: www.insideprivacy.com/covid-19/covid-19-apps-and-websitesthe-pan-european-privacy-preserving-proximity-tracing-initiative-and-guidance-bysupervisory-authorities/ (accessed 27 April 2021).

Crocker, R., Opsahl, K. and Cyphers, B. (2020), “The challenge of proximity apps for COVID-19 contact tracing", Electron. Front. Found.

Davies, B., Innes, M. and Dawson, A. (2018), “An evaluation of South Wales police's use of automated facial recognition", University of Cardiff.

Davis, S. (2020), "Contact tracing apps: extra risks for women and marginalized groups", Health Hum. Rights J.

Eames, K.T.D. (2007), "Contact tracing strategies in heterogeneous populations", Epidemiology and Infection, Vol. 135 No. 3, pp. 443-454.

Fox, J. (2020), "How bad is the coronavirus? Let's run the numbers", Bloomberg.com. 
JICES
19,4

Fussey, P. and Murray, D. (2019), "Independent report on the London Metropolitan Police Service's trial of live facial recognition technology", Human Right Centre, University of Essex, Essex.

Gillmor, D.K. (2020), "Principles for technology-assisted contact-tracing”, ACLU White Pap.

Goode, L. (2018), “App permissions don’t tell us nearly enough about our apps”, Wired, available at: www.wired.com/story/app-permissions/

Holmes, O. (2020), "Israel to track mobile phones of suspected coronavirus cases", Guard. Newsp.

ICO (2019), ICO Investigation into How the Police Use Facial Recognition Technology in Public Places, London.

Leicester City council (2011), Population and CYP in Leicester, Leicester.

Li, J. and Guo, X. (2020), "COVID-19 contact-tracing apps: a survey on the global deployment and challenges".

London Policing Ethics Panel (2019), "London policing ethics panel: final report on live facial recognition".

McLean, A.R. and Anderson, R.M. (1988), "Measles in developing countries. Part II. The predicted impact of mass vaccination", Epidemiology and Infection, Vol. 100 No. 3, pp. 419-442.

MHPRA (2020), "Regulatory status of software (including apps) used in the diagnosis, treatment and management of patients with coronavirus (COVID-19)".

Miltgen, C.L. and Peyrat-Guillard, D. (2014), "Cultural and generational influences on privacy concerns: a qualitative study in seven european countries", European Journal of Information Systems, Vol. 23 No. 2, pp. 103-125.

Novid (2020), available at: www.novid.org/ [WWW Document], Novid (accessed 1 February 2021).

O Nyumba, T., Wilson, K., Derrick, C.J. and Mukherjee, N. (2018), "The use of focus group discussion methodology: insights from two decades of application in conservation", Methods in Ecology and Evolution, Vol. 9 No. 1, pp. 20-32.

ONS (2011), "2011 census".

ONS (2020), Updating Ethnic Contrasts in Deaths Involving the Coronavirus (COVID-19), England and Wales: deaths Occurring 2 March to 28 July 2020, Office of National Statistics, London.

Otu, A., Ahinkorah, B.O., Ameyaw, E.K., Seidu, A.-A. and Yaya, S. (2020), "One country, two crises: what Covid-19 reveals about health inequalities among BAME communities in the United Kingdom and the sustainability of its health system?”, Int. J. Equity Health, Vol. 19, p. 189, doi: 10.1186/s12939-020-01307-z.

PHE (2020a), Disparities in the Risk and Outcomes of COVID-19, Public Health England, London.

PHE (2020b), Beyond the Data: Understanding the Impact of COVID-19 on BAME Groups, Public Health England, London.

Quinn, B. and Perraudin, F. (2020), "London police accused of racial profiling in lockdown searches", Guard, Newsp.

Scassa, T., Millar, J. and Bronson, K. (2020), "Privacy, ethics, and contact-tracing apps".

Simko, L., Chang, J.L., Jiang, M., Calo, R., Roesner, F. and Kohno, T. (2020), "COVID-19 contact tracing and privacy: a longitudinal study of public opinion".

Troncoso, C., Payer, M., Hubaux, J.-P., Salathé, M., Larus, J., Bugnion, E., Lueks, W., Stadler, T., Pyrgelis, A. and Antonioli, D. (2020), "Decentralized privacy-preserving proximity tracing".

UKRI (2021), “COVID-19 and ethnicity: funding six new projects [WWW document]", UK Res. Innov, available at: www.ukri.org/our-work/tackling-the-impact-of-covid-19/understanding-coronaviruscovid-19-and-epidemics/covid-19-and-ethnicity/ (accessed 4 February 2021).

WHO (2020a), "Ethical considerations to guide the use of digital proximity tracking technologies for COVID-19 contact tracing". 
WHO (2020b), "Contact tracing in the context of COVID-19: interim guidance", 10 May 2020.

UK COVID-19

Woolhouse, M. and Donaldson, A. (2001), "Managing foot-and-mouth", Nature, Vol. 410 No. 6828, pp. 515-516.

Yancy, C.W. (2020), “COVID-19 and African Americans”, JAMA, Vol. 323 No. 19, pp. 1891-1892, doi: $10.1001 /$ jama.2020.6548.

Zhang, Z. and Jain, S. (2020), "Mathematical model of Ebola and Covid-19 with fractional differential operators: non-Markovian process and class for virus pathogen in the environment", Chaos Solitons Fractals, Vol. 140, p. 110175, doi: 10.1016/j.chaos.2020.110175.

\section{Further reading}

DHSC (2020), Data Protection Impact Assessment NHS App - Health in Wales, Department of Health and Social Care.

DHSC (2021), The NHS COVID 19 App: Data Protection Impact Assessment, Department of Health and Social Care.

Mason, R. and Sweney, M. (2021), "Thousands could have isolated for no reason due to Covid app error", Guard, Newsp.

NCSC (2020), High Level Privacy and Security Design for the Contact Tracing App, National Cyber Security Centre.

\section{Appendix. Focus group questions}

\section{Themes}

(1) Initial perceptions:

- Could you explain to us your initial perceptions about the contact tracing app?

- Can you explain the reason for your decision to use or not use the app?

(2) Privacy and data protection:

- Do you think the app presents privacy and data protection challenges?

- How do you think these challenges would affect the uptake of the app in the BAME community?

(3) Socio-economic impact:

- What socio-economic impact do you think this technology will have on the BAME community?

- What other challenges/concerns related to culture, health, politics and economy do you think this technology will present?

(4) Final perceptions:

- Following this discussion, do you think you will download/use/continue the use of the contact tracing app?

\section{About the authors}

Simisola Akintoye is the Data Protection Officer for the EU Human Brain Project. She is an international privacy practitioner, data protection consultant and senior lecturer in law at De Montfort University Centre for Law, Justice and Society. Simisola is also a co-convenor of AI in Africa at DMU. Her work involves continuous, up-to-date research in privacy and data protection. She regularly sits on panels involved in critical dialogues on balancing the competing interests of privacy and innovation at national and international levels. She holds a $\mathrm{PhD}$ in law and LLM degrees in International Commercial Law and Business Law and Taxation. Simisola Akintoye is the corresponding author and can be contacted at: simi.akintoye@dmu.ac.uk 
JICES 19,4

George Ogoh is Research Fellow at the Centre for Computing and Social Responsibility (CCSR) at De Montfort University. He has a background in Computing Information Systems and a $\mathrm{PhD}$ in Computing and Social Responsibility. He is currently engaged in ethics-related research for the EU funded Human Brain Project. His research covers a wide range of themes in technology ethics and social responsibility and includes topics on responsible research and innovation (RRI), responsibility by design, responsible innovation, diversity and equality, data governance and data protection.

Zoi Krokida is Lecturer in Law at De Montfort University Leicester. She holds a $\mathrm{PhD}$ in Information Technology Law and Intellectual Property Law from the University of Reading, an LLM with specialization in European Commercial Law from the University of Freiburg and an LLB from Democritus University of Thrace. Her main research interests lie in the field of information technology law, copyright and trade law. In particular, she is interested in ISSPs' regulatory framework, online copyright and trade mark infringements, online defamation, protection of internet users' rights, enhancement of innovation in the digital world and responsible use of AI technology.

Juliana Nnadi is a $\mathrm{PhD}$ candidate at the Centre for Law, Justice and Society (CLJS), Associate Lecturer at De Montfort Law School, Leicester. She is a Fellow of the Institute of Chartered Secretaries and Administrators (ICSA), UK, and associate member of Chartered Institute of Arbitrators (CIArb), UK. Her research interest cuts across electoral democracy and digital technologies particularly, artificial intelligence. Juliana holds an LLB, BL and LLM in law. She is also a Fellow of the Chartered Institute of Secretaries (FCIS) and an Associate with the Chartered Institute of Arbitrators (AClarb).

Damian Eke has experience and expertise on Ethics of Emerging Technologies including artificial intelligence. He is currently a Research Fellow at the Centre for Computing and Social Responsibility at De Montfort University, UK and is the Data Governance Coordinator for the EU Human Brain Project. His research includes work on responsible data governance of biomedical data, Data Ethics and ICT4D.

For instructions on how to order reprints of this article, please visit our website: www.emeraldgrouppublishing.com/licensing/reprints.htm Or contact us for further details: permissions@emeraldinsight.com 